\title{
Early-life experiences altered the maturation of the lateral habenula in mouse models, resulting in behavioural disorders in adulthood
}

\author{
Tomoya Nakamura, PhD; Kohei Kurosaki, MD, Munenori Kanemoto, PhD; \\ Masakiyo Sasahara, MD, PhD; Hiroyuki Ichijo, MD, PhD
}

\begin{abstract}
Background: Abnormally high activity in the lateral habenula causes anxiety- or depression-like behaviours in animal experimental models. It has also been reported in humans that excessive stress in early life is correlated with the onset of psychiatric disorders in adults. These findings raise the question of whether maturation of the lateral habenula is affected under the influence of early-life experiences, which could govern behaviours throughout life. Methods: We examined the maturation of the lateral habenula in mice based on neuronal activity markers and plastic components: Zif268/Egr1, parvalbumin and perineuronal nets. We examined the effect of early-life stress using repeated maternal deprivation. Results: First, we found a transient highly sensitive period of the lateral habenula under stress. The lateral habenula matured through 4 stages: postnatal days 1-9 (P1-9), P10-20, around P35 and after P35. At P10-20, the lateral habenula was highly sensitive to stress. We also observed experience-dependent maturation of the lateral habenula. Only mice exposed to chronic stress from P10-20 exhibited changes specific to the lateral habenula at P60: abnormally high stress reactivity shown by Zif268/Egr1 and fewer parvalbumin neurons. These mice showed anxiety- or depression-like behaviours in the light-dark box test and forced swim test. Limitations: The effect of parvalbumin neurons in the lateral habenula on behavioural alterations remains unknown. It will be important to understand the "sensitive period" of the neuronal circuits in the lateral habenula and how the period P10-20 is different from P9 or earlier, or P35 or later. Conclusion: In mice, early-life stress in the period P10-20 led to late effects in adulthood: hyperactivity in the lateral habenula and anxiety or depression, indicating differences in neuronal plasticity between stages of lateral habenula maturation.
\end{abstract}

\section{Introduction}

The lateral habenula in the dorsal diencephalon is activated by aversive information from the basal ganglia and limbic area, and it inhibits the activity of downstream monoaminergic systems in the midbrain, such as the ventral tegmental area and the dorsal raphe nucleus, which regulate emotion and cognition. ${ }^{1-5}$ It has been suggested that dysfunction of the lateral habenula causes behavioural disorders such as anxiety and depression.

Anxiety-like behaviours and hyperactivity in the lateral habenula have been shown in rats after ethanol withdrawal. ${ }^{6}$ Neurons in the lateral habenula projecting to the ventral tegmental area are activated in learned helplessness animal models of depressive-like disorder, ${ }^{7-9}$ and activation of $\gamma$-aminobutyric acid (GABA)-ergic neurons in the lateral habenula ameliorate depression behaviour. ${ }^{10}$ In humans, hyperactivity of neurons in the lateral habenula has been shown to occur in people with depression. ${ }^{11}$ Thus, it is thought that excessive activity in the lateral habenula is involved in the mechanisms that underlie anxiety and depression.

In epidemiological studies, anxiety- or depression-related disorders are known to be caused by aversive experiences such as excessive stress during childhood in the form of child neglect and abuse in humans. ${ }^{12,13}$ In rodent experiments, anxiety- or depression-like behaviours in adulthood occur after 3 hours of repeated maternal deprivation during postnatal days 1 to 21 (P1-21). ${ }^{14,15}$ These studies often focus on the hypothalamic-pituitary-adrenal system, which acts as a major defence against stress stimulation. ${ }^{16,17}$ On the other hand, it has been reported that 6 hours of repeated maternal deprivation during the period P7-15 results in insufficient $\mathrm{GABA}_{\mathrm{B}}$ receptor signalling, lateral habenula hyperexcitability and depression-like behaviours. ${ }^{18}$

Early-life experiences have also been known to disturb the maturation of neurons and cause functional and structural alterations in the neural circuits throughout life; this property of altering neural circuits is referred to as plasticity. In rodent

Correspondence to: H. Ichijo, Department of Anatomy, Faculty of Medicine, University of Toyama, Sugitani 2630, Toyama, 930-0194, Japan; ichijo@med.u-toyama.ac.jp

Submitted Dec. 9, 2020; Revised Mar. 10, 2021; Accepted Apr. 19, 2021

DOI: 10.1503/jpn.200226 
studies, high neuronal plasticity has been reported to depend on high neuronal activity in early life. ${ }^{19}$ Our previous study shows that the lateral habenula has higher reactivity to stress in early life than in adulthood, using the expression of an immediate-early gene (Zif268/Egr1), a reliable marker for increased neuronal activity. ${ }^{20}$ However, the maturation of the lateral habenula and its relationship with plasticity have not been described in detail.

We investigated the course of maturation in terms of neuronal plastic markers and sensitivity to stresses; experiencedependent changes in neuronal plastic markers and sensitivity to stress; and experience-dependent plastic changes in behaviour. Stressful experiences in early life disturbed the maturation of the lateral habenula, resulting in hyperactivity in the lateral habenula and anxiety and depression in adulthood.

\section{Methods}

\section{Animals}

Information about experimental animals (C57BL/6J, GAD1GFP knock-in mice, ${ }^{21}$ GAD2-mCherry mice, ${ }^{22,23}$ GAD2-IRESCre mice and R26R-H2B-mCherry mice) and their care is provided in Appendix 1, available at jpn.ca/200226-a1. We used GAD2-mCherry mice for the experiments described in Appendix 1, Figures S1D and S2; we used GAD1-GFP knockin mice in the experiment described in Appendix 1, Figure S1E; and we used C57BL/6J mice in the other experiments.

This study was performed in strict accordance with the guidelines for the care and use of laboratory animals approved by the University of Toyama and the US National Institutes of Health Guide for the Care and Use of Laboratory Animals, ${ }^{24}$ and it was approved by the ethics committee for animal experiments at the University of Toyama (licence numbers A2016MED-38 and A2019MED-34).

\section{Immunohistochemistry and lectin histochemistry}

Mice were transcardially perfused with phosphate-buffered saline (PBS) and then with 3.7\% formaldehyde (Wako) in PBS under deep anesthesia with pentobarbital $(50 \mathrm{mg} / \mathrm{kg}$ body weight, i.p.; Kyoritsu Seiyaku). Perfusion was completed 30 minutes before the expression of Zif268/Egr1 started, so the influence of general stress during the experiments was excluded. Brains were dissected and kept overnight in the same fixative at $4^{\circ} \mathrm{C}$. After washing in PBS, coronal sections of $70 \mu \mathrm{m}$ thickness were cut using a vibratome (VT1000S; Leica Microsystems). Along the length of the lateral habenula, from the anterior (at -1.22 from the bregma) to the posterior (at -2.06 from the bregma), we collected 14 serial sections. ${ }^{25}$

The serial sections were divided into odd- and evennumbered sections. Alternate sections were incubated with primary antibodies in $0.5 \%$ Triton $\mathrm{X}-100$ in PBS (PBST) with $5 \%$ normal goat serum (16210064; Thermo Fisher Scientific; PBSTN) or biotinylated Wisteria floribunda agglutinin (WFA) lectin (B-1355; Vector) in PBST for 3 days. The sections were incubated in the appropriate biotinylated secondary antibody for 2 hours, washed and incubated for 2 hours in ABC reagent (PK-6100; Vector). Signals were visualized using a Metal Enhanced DAB Substrate Kit (34065; Thermo Fisher Scientific).

For double immunohistochemistry, the remaining sections were incubated for 6 days with primary antibody in 3\% bovine serum albumin (9048-46-8; Wako) in PBST, washed, incubated with appropriate fluorescent-secondary antibodies or streptavidin, and counterstained with 4',6-diamidino2-phenylindole (D9542; Sigma-Aldrich).

Primary antibodies (anti-aggrecan, anti-GABA, antimCherry, anti-NeuN, anti-parvalbumin, anti-Zif268/Egr1), biotinylated WFA lectin and secondary antibodies are described in detail in Appendix 1. We performed GABA immunohistochemistry using a STAINperfect Immunostaining Kit (ImmuSmol). We obtained images using a confocal laser scanning microscope (LSM780; Zeiss); image acquisition procedures are described in Appendix 1.

\section{Single maternal deprivation and single restraint stress}

For single maternal deprivation, each pup was separated from its dam and placed in a transparent plastic box $(15 \mathrm{~cm}$ length $\times 21 \mathrm{~cm}$ width $\times 13 \mathrm{~cm}$ height) with fresh animal bedding at room temperature $\left(22-25^{\circ} \mathrm{C}\right)$ for 2 hours $(1000$ $1200 \mathrm{~h})$. Then, they were perfusion-fixed.

For single restraint stress, mice were restrained for $60 \mathrm{~min}-$ utes in $50 \mathrm{~mL}$ tubes with air holes (339652; Thermo Fisher Scientific) at room temperature $\left(22-25^{\circ} \mathrm{C}\right)$. Then, mice were kept for 60 minutes in their home cage until the number of Zif268/Egr1-positive cells reached its maximum in the lateral habenula after stress. ${ }^{20}$ Following this, mice were perfusionfixed. We assessed neuronal activity using the density of Zif268/Egr1-positive cells in the lateral habenula and the dentate gyrus.

\section{Repeated maternal deprivation and repeated restraint stress}

To model chronic stress in early life, maternal deprivations were repeated for 3 hours per day between 0800 and $1200 \mathrm{~h}$ during the periods $\mathrm{P} 1-9$ (at $25^{\circ} \mathrm{C}$ ) or P10-20 (at $22-25^{\circ} \mathrm{C}$ ). We analyzed the immediate effects of repeated maternal deprivation on P20. After repeated maternal deprivation, the mice were weaned on P30. We analyzed the late effects of maternal deprivation on a day between P60 and P87; mice were subjected to behavioural tests or perfusion-fixed 1 hour after treatment. To model chronic stress in adulthood, restraint stress was repeated for 1 hour per day between 0800 and 1200 h from P36 to P45.

\section{Plasma corticosterone concentration}

To minimize the influence of circadian fluctuations, blood samples were collected between 1130 and $1230 \mathrm{~h}$ under deep anesthesia from the left ventricle with a 26-gauge needle and a syringe washed with heparin (5000 units $/ 5 \mathrm{~mL}$; Mochida Pharmaceutical Co.) in an ice-cooled tube. Samples were centrifuged $\left(3000 \mathrm{~g}, 10\right.$ minutes, $\left.4^{\circ} \mathrm{C}\right)$ and the plasma was stored 
at $-80^{\circ} \mathrm{C}$. We measured plasma corticosterone concentrations using an AssayMax Corticosterone ELISA kit (Assaypro LLC). Absorbance at $450 \mathrm{~nm}$ was read on a microplate reader (Multiskan FC; Thermo Fisher Scientific). Means were calculated for triplicate sets of standards and samples.

\section{Behavioural tests}

In the light-dark box test, the light (200 lux) and dark apparatus consisted of 2 identically sized compartments $(15 \mathrm{~cm}$ length $\times 15 \mathrm{~cm}$ width $\times 30 \mathrm{~cm}$ height) connected by a small opening $(4 \mathrm{~cm} \times 4 \mathrm{~cm})$. The apparatus was wiped with $70 \%$ ethanol and air-dried. First, the mice were placed in the dark compartment. Using a digital camera in the light compartment (c920; Logitech), the mice were recorded for 5 minutes, and time spent in the light compartment was measured. Time spent in the dark compartment was calculated.

Forced swim tests were performed 10 days after the lightdark box test. A cylinder $(22 \mathrm{~cm}$ diameter $\times 22 \mathrm{~cm}$ height) was filled with water up to a height of $15 \mathrm{~cm}\left(25-28^{\circ} \mathrm{C}\right)$. Mice were placed in the cylinder one at a time, and their activities were recorded using the digital camera for 10 minutes. Their positions were traced using Motr $^{26}$ and analyzed using a custom-written MATLAB script. Details of the behavioural tests are described in Appendix 1.

To avoid stress stimulation before the behavioural tests, we took strict precautions. The time spent in the behavioural tests was short: 5 minutes for the light-dark box test and 10 minutes for the forced swim test. Plasma corticosterone concentration was not thought to be related to the outcomes of the behavioural tests in the present study, because plasma corticosterone concentrations increased gradually, peaked after 60 minutes and subsided after 150 minutes of acute stress. ${ }^{27}$

\section{Statistical analysis}

Data were expressed as mean \pm standard error of the mean. They were analyzed using the Kruskal-Wallis rank-sum test followed by the Steel-Dwass multiple comparison test; a simple regression test; Student $t$ test; 1 -way analysis of variance (ANOVA) followed by a Tukey honestly significant difference (HSD) test; or 2-way ANOVA after the $F$ test or Levene test. Statistical significance was set at $p<0.05$. Analyses were performed using JMP Pro 14.2 (SAS Institute) or Excel (Microsoft).

\section{Results}

Maturation of the lateral habenula from early life to adulthood: parvalbumin neurons and perineuronal nets

In the lateral habenula, we measured the density of parvalbumin (PV) neurons and the percentage area covered by perineuronal nets (PNNs) on a day between P10 and P82 (Figure 1A). In the control group, the density of PV neurons was significantly different by age $\left(\chi^{2}=7.99, p=0.018\right.$, Kruskal-Wallis rank-sum test; $F=0.59, p=0.57$, Levene test). The density of PV neurons was higher at P60 than that at P20 ( $p=0.033$, Steel-Dwass multiple comparison test; Figure 1B to D). Using a simple regression test, we found that the density of PV neurons was positively correlated with age from P20 to P60 $\left(r^{2}=0.46, p=0.002\right.$; Figure $\left.1 \mathrm{E}\right)$.

The percentage area covered by PNNs was significantly different by age $\left(\chi^{2}=20.32, p<0.001\right.$, Kruskal-Wallis ranksum test; $F=9.22, p<0.001$, Levene test). Percentage areas were higher in adulthood than in early life and adolescence: higher at P60-82 than at P35-40 ( $p=0.046)$; higher at P35-40 than at P20 ( $p=0.041)$; and higher at P20 than at

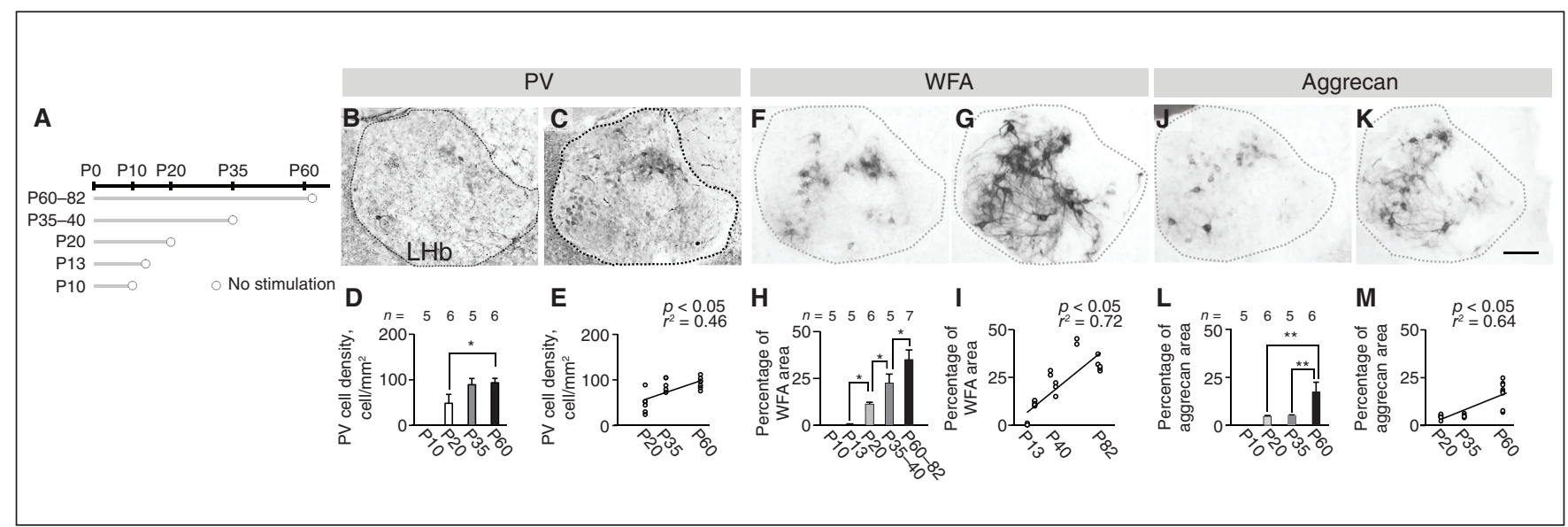

Figure 1: Maturation of PV neurons and PNNs in the LHb. (A) Schematic diagram of experiments. (B and C) PV neurons at P20 and P60. (D) Cell densities at each postnatal day. (E) Correlation between postnatal day and cell density is shown using a simple regression test. ( $F$ and G) WFA lectin staining at P20 and P60. (H) Percentage of WFA area at each postnatal day. (I) Correlation between postnatal day and percentage of WFA lectin staining area is shown using a simple regression test. ( $\mathrm{J}$ and $\mathrm{K}$ ) Anti-aggrecan immunoreactivities at P20 and P60. (L) Percentage of aggrecan area at each postnatal day. (M) Correlation between postnatal day and percentage of anti-aggrecan immunoreactivity area using a simple regression test. Scale bar $=100 \mu \mathrm{m}$. Error bars represent standard error of the mean. The square correlation coefficient $(r)$ and $p$ values of the slope are shown. ${ }^{*} p<0.05$, ${ }^{* *} p<0.01$, Steel-Dwass multiple comparison test. LHb $=$ lateral habenula; $\mathrm{P}=$ postnatal day; PNN = perineuronal nets; PV = parvalbumin; WFA = wisteria floribunda agglutinin. 
P13 ( $p=0.041$ ) based on WFA lectin (Steel-Dwass multiple comparison test; Figure $1 \mathrm{~F}$ to $\mathrm{H}$ ) and anti-aggrecan $\left(\chi^{2}=13.34, p=0.001\right.$, Kruskal-Wallis rank-sum test; $F=$ $6.17, p<0.001$, Levene test). They were also higher at P60 than at P20 $(p=0.004)$ and P35 ( $p=0.012$; Steel-Dwass multiple comparison test; Figure $1 \mathrm{~J}$ to L). Using a simple regression test, we found that the percentage area covered by PNNs was positively correlated with age from P13 to P82 (WFA, $r^{2}=0.72, p<0.001$; aggrecan, $r^{2}=0.64, p<0.001$; Figure $1 \mathrm{I}$ and $\mathrm{M}$ ).

We scrutinized PV neurons using reliable markers to verify their detailed character in control mice at P60. The PV neurons were positive for $\mathrm{NeuN}(99.9 \pm 0.1 \%)$, anti-GABA $(56.4 \pm 6.4 \%)$ and GAD2-mCherry $(4.8 \pm 0.9 \%)$, and were surrounded by PNNs $(79.6 \pm 1.9 \%$ with WFA lectin staining and $86.1 \pm 0.7 \%$ with anti-aggrecan immunostaining; Appendix 1, Figure S1). We observed no GAD1-positive cells in the lateral habenulas of GAD1-GFP knock-in mice (Appendix 1, Figure S1E), indicating that PV neurons in the lateral habenula contained GABA without expression of GAD2 or GAD1. The immunostaining signals for anti-
GABA antibodies were validated by colocalization with GAD2-mCherry signals in the cerebral cortex and the lateral habenula (Appendix 1, Figure S2).

In control mice, the number of PV neurons at P60 was $195.28 \pm 25.76$ cells in single staining (mean \pm standard deviation; $n=7)$ and $165.5 \pm 43.24$ cells in double staining $(n=6)$, suggesting that single staining was more accurate, with a smaller standard deviation. We used single staining in the following experiments.

Maturation of the lateral habenula from early life to adulthood: neuronal reactivity during stress

We examined activation of the lateral habenula after single maternal deprivation or single restraint stress from early life to adulthood. To determine neuronal activity without stimulation, we measured the density of Zif268/Egr1 immunopositive cells (Zif268/Egr1 cells) on a day between P9 and P70 (Figure 2A). Densities were significantly different by age $\left(\chi^{2}=26.27, p<0.001\right.$, Kruskal-Wallis rank-sum test; $F=4.56$, $p=0.003$, Levene test): higher at P20-21 ( $p<0.05)$, P35 $(p<$

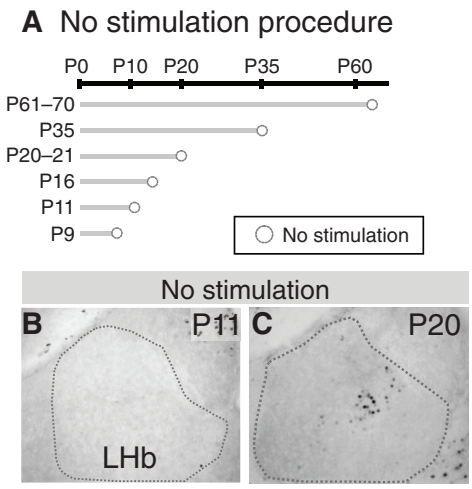

D

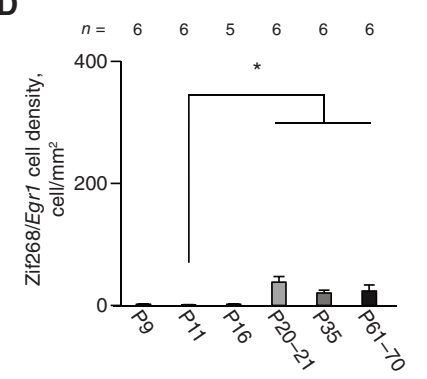

E Single maternal deprivation
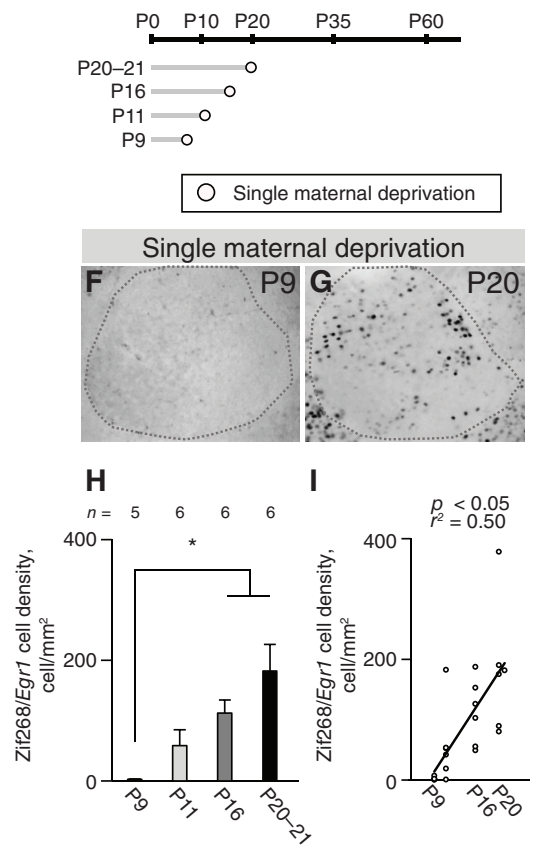

J Single restraint stress

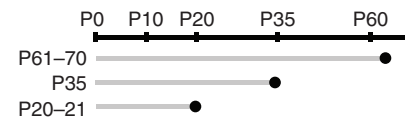

Single restraint stress
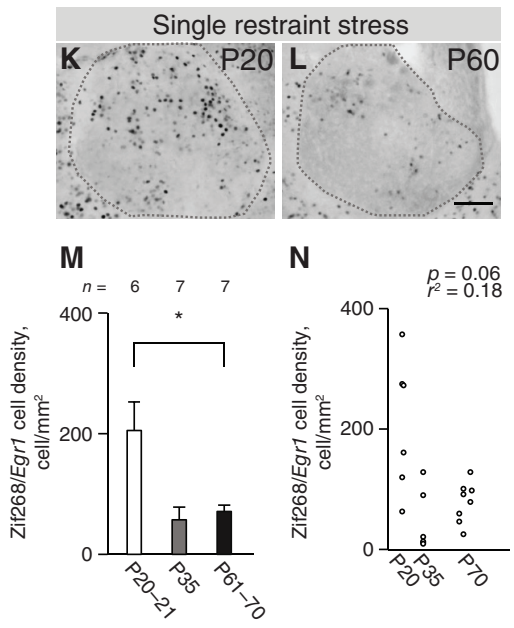

$\mathbf{N}$

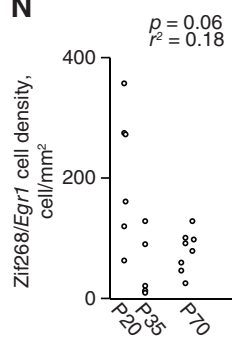

Figure 2: Postnatal changes in neuronal reactivity in response to single maternal deprivation or single restraint stress during maturation of the LHb. (A) Schematic diagram of experiments without stress stimulation. (B and C) Zif268/Egr1 immunopositive cells at P11 and P20 with no stimulation. (D) Cell densities at each postnatal day. (E) Schematic diagram of experiments with single maternal deprivation. ( $F$ and $G$ ) Zif268/ Egr1 immunopositive cells at P9 and P20 after single maternal deprivation. (H) Cell densities at each postnatal day. (I) Correlation between postnatal day and cell density after single maternal deprivation using a simple regression test. $(\mathrm{J})$ Schematic diagram of experiments with single restraint stress. (K and L) Zif268/Egr1 immunopositive cells at P20 and P60 after single restraint stress. (M) Cell densities at each postnatal day. (N) Using a simple regression test, we observed no correlation between postnatal day and Zif268/Egr1 immunopositive cell density after single restraint stress. Scale bar $=100 \mu \mathrm{m}$. Error bars represent standard error of the mean. The square correlation coefficient $(r)$ and $p$ values of the slope are shown. ${ }^{*} p<0.05$, Steel-Dwass multiple comparison test. $\mathrm{LHb}=$ lateral habenula; $\mathrm{P}=$ postnatal day. 
0.05) and P61-70 ( $p<0.05)$ than at P11 (Figure 2B to D). We found no significant differences between other age groups.

To determine neuronal activity in response to acute stress stimulation, we measured the density of Zif268/Egr1 cells after single maternal deprivation as an acute stress stimulation on a day between P9 and P21 (Figure 2E), or as a single restraint stress on a day between P20 and P70 (Figure 2J). We did this because single maternal deprivation was not suitable after weaning. After single maternal deprivation, densities were significantly different by age from P9 to P21 $\left(\chi^{2}=14.29, p=0.003\right.$, Kruskal-Wallis rank-sum test; $F=$ $7.72, p=0.19$, Levene test): higher at P16 $(p=0.041)$ and P20-21 ( $p=0.041)$ than at P9 (Steel-Dwass multiple comparison test; Figure $2 \mathrm{~F}$ to $\mathrm{H}$ ). We found no significant differences between the other age groups. After single restraint stress, densities were significantly different between ages $\left(\chi^{2}=7.71, p=0.021\right.$, Kruskal-Wallis rank-sum test; $F=$ $11.32, p<0.001$, Levene test): lower at P61-70 than at P20-21 $(p=0.048$, Steel-Dwass multiple comparison test; Figure $2 \mathrm{~K}$ to $\mathrm{M})$. In a simple regression test, density after single maternal deprivation was positively correlated with P9-21 $\left(r^{2}=\right.$ $0.50, p=0.022$; Figure 2I), but density after single restraint stress was not significantly correlated with P20-70 $\left(r^{2}=0.18\right.$, $p=0.06$; Figure 2N).

At P20-21, the densities of Zif268/Egr1 cells were significantly different between the single maternal deprivation, single restraint stress and no stimulation groups $\left(\chi^{2}=10.7, p=\right.$ 0.005 , Kruskal-Wallis rank-sum test; $F=3.6, p>0.05$, Levene test); densities after the single maternal deprivation and single restraint stress tests were significantly higher than with no stimulation (single maternal deprivation, $p=0.014$; single restraint stress, $p=0.022$; Steel-Dwass multiple comparison test). Moreover, the densities after single maternal deprivation and single restraint stress were not significantly different at P20-21 ( $p=0.99$, Steel-Dwass multiple comparison test), indicating that the effects of these stimulations on the lateral habenula were similar. Therefore, these stimulations were suitable for comparing stress responses in the lateral habenula from early life to adulthood. In the adult lateral habenula after single restraint stress (acute stress), $97.4 \pm 8.4 \%$ of the Zif268/Egr1 cells were negative for PV immunoreactivity $(n=5)$; thus, most of the cells activated by stress were not PV neurons at P60 (Appendix 1, Figure S3).

We found almost no Zif268/Egr1 cells at P9, P11 or P16 without stress stimulation, and at $\mathrm{P} 9$ with maternal deprivation. In these conditions, the densities of Zif268/Egr1 cells showed very small variances. Because variances were unequal between groups, nonparametric tests were required, but there is no statistical method for a 2-way layout design in a nonparametric test. At P61-70, we used statistical tests for independent groups; the densities of Zif268/Egr1 cells after single restraint stress were higher than after no stimulation $\left(t_{11}=3.19, p=0.009\right.$, Welch $t$ test; $p=0.68, F$ test $)$. We observed that with single maternal deprivation, Zif268/Egr1 cell densities at P11 and P16 were explicitly higher than cell densities in mice without stimulation. These results indicate that neuronal activity in the lateral habenula is high with stress stimulation after P11.

\section{Alteration in lateral habenula maturation with early-life stress}

We evaluated neuronal reactivity to single restraint stress in the adult lateral habenula in the control (no chronic stress), repeated maternal deprivation (P1-9), repeated maternal deprivation (P10-20) and repeated restraint stress (P36-45) groups by examining the density of Zif268/Egr1 cells after single restraint stress (Figure 3A) or no stimulation (Figure 3B). At $\mathrm{P} 60-70$ in the lateral habenula, densities after single restraint stress were significantly different between groups $\left(F_{3,23}=6.59 ; p=0.002,1\right.$-way ANOVA; $F=2.1, p=0.13$, Levene test): higher in the repeated maternal deprivation (P10-20) group than in the control $(p=0.008)$, repeated maternal deprivation $(\mathrm{P} 1-9 ; p=0.037)$ and repeated restraint stress groups $(p=0.004)$, respectively (Tukey HSD test; Figure $3 C$ to $G)$. Without stimulation, densities were not significantly different between groups $\left(F_{3,20}=2.71, p=0.07\right.$, in 1 -way ANOVA; $F=1.05, p=0.39$, Levene test; Figure $3 \mathrm{H})$. To understand the effect of repeated maternal deprivation (P10 20) on major stress-related areas, we measured the densities of Zif268/Egr1-positive neurons in the dentate gyrus and basolateral amygdala. In the dentate gyrus, densities after single restraint stress were not significantly different between the control and repeated maternal deprivation (P10-20) groups $\left(t_{13}=0.93, p=0.37\right.$, Student $t$ test; $p=0.18, F$ test; Figure 3I to K). In the basolateral amygdala, densities after single restraint stress were not significantly different from those with no stimulation $\left(t_{10}=0.19, p=0.85\right.$, Student $t$ test; $p=0.92, F$ test; Appendix 1, Figure S4). Although it is well known that the basolateral amygdala shows neuronal activity in response to stress, we did not observe neuronal stress reactivity based on the density of Zif268/Egr1 cells.

At P60-67, plasma corticosterone concentration after single restraint stress was significantly higher than without stimulation (main effect of stress; $F_{1,28}=59.11, p<0.001$, 2-way ANOVA; Figure 3L). However, we found no significant difference in plasma corticosterone concentration between the control, repeated maternal deprivation (P1-9) and repeated maternal deprivation (P10-20) groups after single restraint stress or no stimulation (main effect of treatment; $F_{2,28}=2.40, p=0.11$; interaction, $F_{2,28}=0.12, p=0.88$, 2-way ANOVA; Figure 3L). These findings showed that in the repeated maternal deprivation (P10-20) group, neuronal reactivity in the lateral habenula after single restraint stress was higher than in other groups; however, general stress responses were similar across groups.

To understand the relationship between higher neuronal reactivity and the expression of neuronal maturation markers in the adult lateral habenula after repeated maternal deprivation (P10-20), we focused on PV neurons and PNNs. The density of PV neurons in the lateral habenula at P60-70 was significantly different between groups $\left(F_{3,22}=9.13, p<0.001\right.$, 1-way ANOVA; $F=0.81, p=0.49$, Levene test): we observed significantly fewer PV neurons in the lateral habenula in the repeated maternal deprivation (P10-20) group than in the control group $(p=0.002)$, the repeated maternal deprivation (P1-9) group $(p=0.003)$ or the repeated restraint stress group ( $p=0.002$, Tukey HSD test; Figure 4A to E). In contrast, the densities of PV neurons were not significantly different 


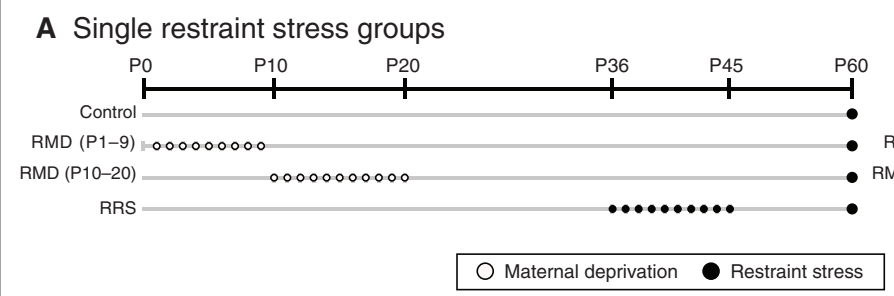

B No stimulation groups

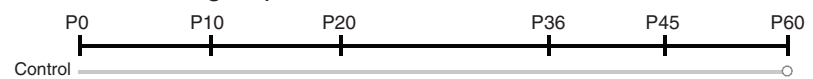

RMD (P1-9) 10000000000

$\mathrm{RMD}(\mathrm{P} 10-20)$
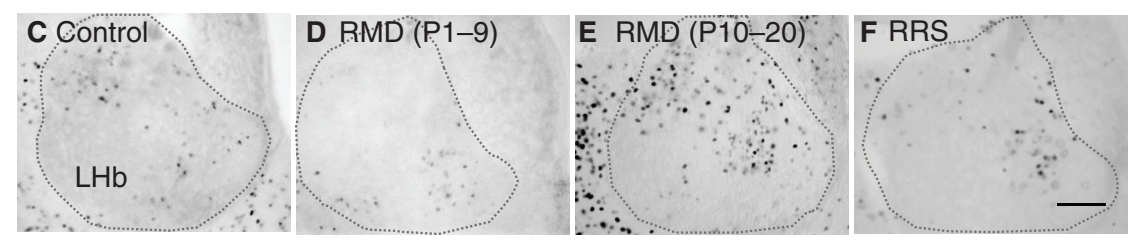

G Single restraint stress $\mathbf{H}$ No stimulation

RRS -

Maternal deprivation

- Restraint stress $\bigcirc$ No stimulation

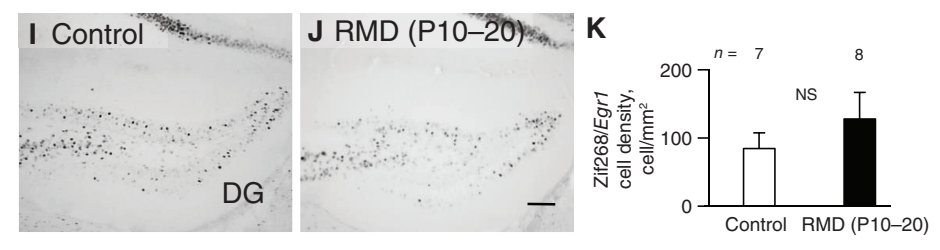

L Corticosterone
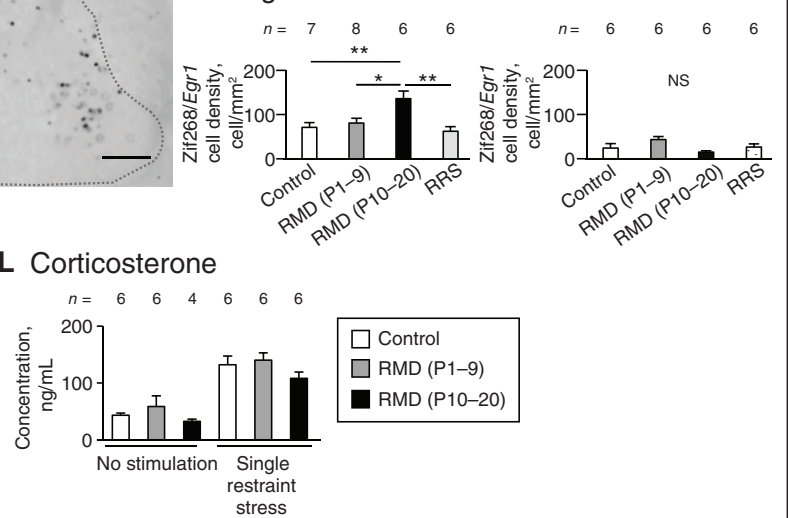

Figure 3: Neuronal hyperreactivity to single restraint stress in the adult LHb under the influence of early-life stress at P10-20. (A and B) Schematic diagram of experiments with single restraint stress and no stimulation. In the LHb, Zif268/Egr1 immunopositive cells in response to single restraint stress are shown in the (C) control, (D) RMD (P1-9), (E) RMD (P10-20) and (F) RRS groups. (G and H) Zif268/Egr1 immunopositive cell densities are compared between the groups after single restraint stress and no stimulation. (I and J) In the dentate gyrus, Zif268/ Egr1 immunopositive cells after single restraint stress in the control and RMD (P10-20) groups. (K) Comparison of Zif268/Egr1 immunopositive cell densities. (L) After single restraint stress or no stimulation, comparison of plasma corticosterone concentrations for the control, RMD (P1-9) and RMD (P10-20) groups at P60-67. Scale bar $=100 \mu \mathrm{m}$. Error bars represent standard error of the mean. ${ }^{*} p<0.05,{ }^{* *} p<0.01$, Tukey honestly significant difference test. $\mathrm{DG}=$ dentate gyrus; $\mathrm{LHb}=$ lateral habenula; $\mathrm{NS}=$ not significant; $\mathrm{P}=$ postnatal day; RMD = repeated maternal deprivation; RRS = repeated restraint stress.

between the control and repeated maternal deprivation (P1020) groups in the dentate gyrus $\left(t_{12}=0.60, p=0.57\right.$, Student $t$ test; $p=0.53, F$ test; Figure $4 \mathrm{~F}$ to $\mathrm{H}$ ) or basolateral amygdala $\left(t_{12}=0.98, p=0.35\right.$, Student $t$ test; $p=0.73, F$ test; Figure $4 \mathrm{I}$ to $\mathrm{K})$. At $\mathrm{P} 60-70$ in the lateral habenula, the percentage area covered by PNNs (WFA and aggrecan) did not differ significantly between the control and repeated maternal deprivation (P10-20) groups (WFA: $t_{12}=0.13, p=0.58$, Student $t$ test and $p=0.90, F$ test; aggrecan: $t_{12}=0.37, p=0.79$, Student $t$ test, and $p=0.72, F$ test; Appendix 1, Figure S5).

In the course of lateral habenula maturation at P20 (Appendix 1, Figure S6A), the density of PV neurons was not significantly different between the control and repeated maternal deprivation (P10-20) groups $\left(t_{10}=0.020, p=0.99\right.$, Student $t$ test; $p=0.98, F$ test; Appendix 1, Figure S6B to D). However, the percentage area covered by WFA-positive PNNs was significantly higher in the repeated maternal deprivation (P10-20) group than the control group $\left(t_{10}=1.99\right.$, $p<0.001$, Student $t$ test; $p=0.07$, in $F$ test; Appendix 1, Figure $\mathrm{S} 6 \mathrm{E}$ to G). The density of WFA-positive cells was not significantly different between groups $\left(t_{10}=2.10, p=0.16\right.$, Student $t$ test; $p=0.053, F$ test; Appendix 1, Figure S6H). Repeated maternal deprivation (P10-20) had specific effects on the lateral habenula, resulting in fewer PV neurons and higher neuronal reactivity at $\mathrm{P} 60-70$ after the enlargement of PNNs at P20. Thus, lateral habenula maturation was altered under the influence of early-life stress at P10-20.

\section{Behavioural disorders under the influence of early-life stress}

We examined behaviours under the influence of early-life stress using the light-dark box test and the forced swim test. We conducted the light-dark box test on a day between P70 and 77; 10 days later, we conducted the forced swim test. In the light-dark box test, mice from the repeated maternal deprivation (P10-20) group spent a longer time in the dark compartment than mice from the control group $\left(t_{28}=3.34, p=\right.$ 0.002 , Student $t$ test; $p=0.66, F$ test; Figure 5A), showing anxiety-like behaviours. As well, in the forced swim test, immobilization time was significantly higher in the repeated maternal deprivation (P10-20) group than in the control group $\left(t_{28}=2.17, p=0.038\right.$, Student $t$ test; $p=0.65, F$ test; Figure $5 \mathrm{~B})$, indicating that mice in the repeated maternal deprivation (P10-20) group showed depression-like 


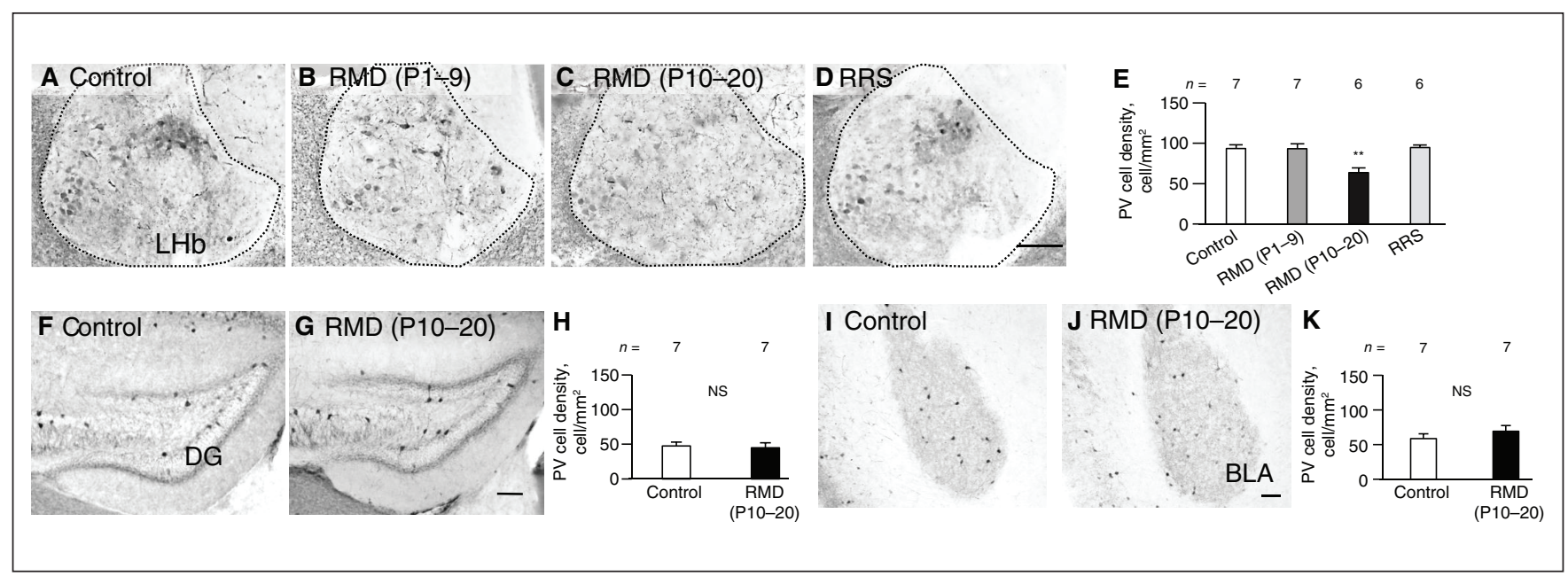

Figure 4: Reduced density of PV neurons in the adult LHb after early-life stress during P10-20. (A to D) In the LHb, PV neurons are shown for the control, RMD (P1-9), RMD (P10-20) and RRS (P36-45) groups. (E) PV cell densities compared between groups. (F and G) In the dentate gyrus, PV neurons are shown for the control and RMD (P10-20) groups. (H) PV cell densities compared between groups. (I and J) PV neurons in the basolateral amygdala. (K) PV cell densities compared between groups. Scale bar $=100 \mu \mathrm{m}$. Error bars represent standard error of the mean. ${ }^{* *} p<0.01$, Tukey honestly significant difference test. BLA = basolateral amygdala; $\mathrm{DG}=$ dentate gyrus; LHb $=$ lateral habenula; $\mathrm{NS}=$ not significant; $\mathrm{P}=$ postnatal day; $\mathrm{PV}=$ parvalbumin; RMD = repeated maternal deprivation; $\mathrm{RRS}=$ repeated restraint stress .

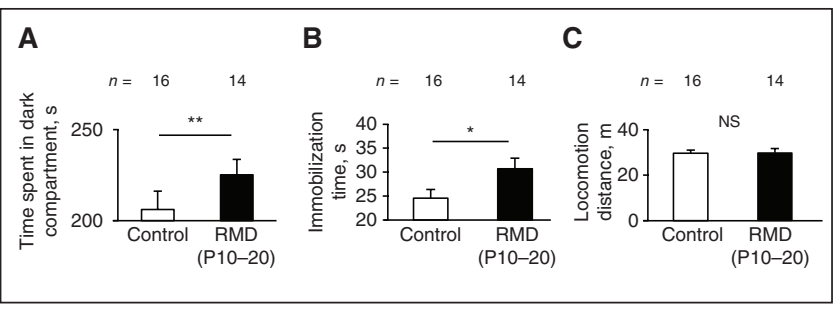

Figure 5: Anxiety/depression-like behaviours in adults exposed to early-life stress. (A) In the light-dark box test, we compared time spent in the dark compartment between the control and RMD (P1020) groups. ( $B$ and $C$ ) In the forced swim test, we compared immobilization times and locomotion distances between the control and RMD (P10-20) groups. Error bars represent standard error of the mean. ${ }^{*} p<0.05,{ }^{* *} p<0.01$, Student $t$ test. NS $=$ not significant; $\mathrm{P}=$ postnatal day; $\mathrm{RMD}=$ repeated maternal deprivation.

behaviours. We found no significant difference in total locomotion distances between the control and repeated maternal deprivation (P10-20) groups $\left(t_{28}=0.099, p=0.92\right.$, Student $t$ test; $p=0.37, F$ test; Figure $5 \mathrm{C}$ ), showing that general locomotor activity was not impaired in the repeated maternal deprivation (P10-20) group. These results indicated that early-life stress during P10-20 elicited anxiety- and depression-like behavioural disorders in adults.

\section{Discussion}

This study revealed the influence of aversive experiences in early life on maturation of the lateral habenula and behaviours during adulthood, indicating experience-dependent mechanisms that modulate lateral habenula neuronal circuits.

The lateral habenula matures gradually; the 4 stages are recognized by neuronal activity, PV neurons and PNNs. In the first stage in mice, around P10, the lateral habenula is immature: we observed no or low neuronal activity and detected neither PV neurons nor PNNs (Figures 1 and 2). The second stage occurs around P20, during which we observed large spontaneous activity, maximum reactivity to single restraint stress and single maternal deprivation, and the appearance of PV neurons and PNNs (Figures 1 and 2). From the first stage to the second stage, neuronal reactivity gradually increased in response to single maternal deprivation (Figure $2 \mathrm{H}$ and I). The third stage occurs around P35, during which we observed low spontaneous activity and weaker reactivity to single restraint stress (Figure 2M). The density of PV neurons and area covered by PNNs increased, indicating that the lateral habenula had functionally matured (Figure 1). The fourth stage occurs after P35; during this stage, the area covered by PNNs exhibited additional expansion, indicating further maturation (Figure $1 \mathrm{~F}$ to $M)$. These results showed that there is a transient, highly sensitive period of neuronal reactivity under stress between P11 and P20, because the mild single stress treatment (single maternal deprivation or single restraint stress) induced intense neuronal activity comparing the period outside P11-20. Moreover, there is an inverse relationship between the transient highly sensitive period of neuronal reactivity and neuronal maturation markers (PV and PNNs) in development. By obtaining information about the stages of maturation in detail, we established the optimal timing for experiments on lateral habenula plasticity under emotional experiences in early life.

Early-life experiences disturb the maturation of neurons and subsequently cause plasticity - alterations in functional and structural neural circuits throughout life. For example, in the visual system, PV neurons as inhibitory interneurons in the corte ${ }^{28-30}$ restrict the plasticity of local circuits as a result 
of experiences during maturation, and this consequently influences higher function. ${ }^{31-33}$

In the present study, we showed PV neurons and PNNs in the lateral habenula as neuronal components of plasticity resulting from experiences in early life. In mice subjected to early-life stress at P10-20 (repeated maternal deprivation [P10-20]), enlargement of the PNNs was shown by WFA lectin staining (which recognizes $\mathrm{N}$-acetylgalactosamine) but not by anti-aggrecan immunostaining (Appendix 1, Figure S6). Because PNNs are an extracellular matrix composed mainly of proteoglycans (hybrid molecules between core proteins and glycosaminoglycan chains), ${ }^{34}$ these results indicate that the glycosaminoglycan chains of PNNs increased. The glycosaminoglycan chains of PNNs on P20 were thought to be modified in an experience-dependent manner by chronic stresses in early life. However, at P60-70, the area of PNNs recognized by WFA in the control group overtook that of the repeated maternal deprivation (P10-20) group (Appendix 1, Figure S5). At P60-70, we found fewer PV neurons with significantly higher neuronal reactivity, followed by anxiety- or depression-like behaviours at P70-87 (Figures 3, 4 and 5). Thus, after repeated maternal deprivation (P10-20), maturation of the lateral habenula was impaired gradually from P20 to P60. Early-life stress induced late effects in the adult lateral habenula, showing experiencedependent plastic changes.

We further characterized PV neurons in the adult lateral habenula. Although about $80 \%$ of PV neurons in the lateral habenula were surrounded by PNNs (Appendix 1, Figure S1F and G), more than $40 \%$ are GABA-negative and express little GAD1 and GAD2 (Appendix 1, Figure S1C, D, E and G). Webster and colleagues ${ }^{35}$ have reported finding GABApositive and glutamatergic PV neurons, whose optogenetic activation elicited inhibitory and excitatory responses in the lateral habenula. That study and the present study showed that PV neurons in the lateral habenula are a heterogeneous population consisting of GABAergic and glutamatergic neurons; therefore, the roles of the PV neurons in the lateral habenula are thought to be different from in the cortex.

Plasma corticosterone concentration is well established as a biomarker of general stress responses in the hypothalamicpituitary-adrenal system. Plasma corticosterone concentrations after single restraint stress on P60 were similar between the control, repeated maternal deprivation (P1-9) and repeated maternal deprivation (P10-20) groups (Figure 3L); thus, general stress responses in the adults were similar between groups, although after P10 stress stimulations in earlylife increase plasma corticosterone concentration. ${ }^{17,36}$ The dentate gyrus and basolateral amygdala are known to be activated by stress, and these plastic changes are correlated with anxiety- or depression-like behaviours. ${ }^{37-41}$ However, we found no effect on neuronal activity as observed by Zif268/ Egr1 cells in the dentate gyrus and PV neurons in the dentate gyrus and basolateral amygdala in adults from the repeated maternal deprivation (P10-20) group (Figure 3K, $4 \mathrm{H}$ and $4 \mathrm{~K}$ ). In contrast, in adults in the repeated maternal deprivation (P10-20) group, we observed behavioural changes (anxiety and depression) and elevated neuronal reactivity in the lateral habenula (Figure 3, 4 and 5). These results indicated that the anxiety- and depression-like behaviours in the repeated maternal deprivation (P10-20) group developed through alteration of neuronal reactivity in the lateral habenula but not through alterations in the hypothalamic-pituitaryadrenal system, dentate gyrus or basolateral amygdala. Thus, the effect of early-life stress was specific to the lateral habenula. Furthermore, stresses outside P10-20 (repeated maternal deprivation [P1-9] and repeated restraint stress [P36-45]) did not affect maturation of the lateral habenula, even though the intensity of these stimulations were thought to be comparable, because single maternal deprivation and single restraint stress at P20 induced activations with a similar number of Zif268/Egr1 cells in the lateral habenula. These results show that the effect of early-life stress was specific to P10-20. Although many previous studies about early-life stress have focused on P1-9 and P2-12, the effects on behavioural disorders of stress during these periods varied between studies. ${ }^{16,17}$ The present study revealed specificity in P10-20.

As well, P10-20 has been reported as a "sensitive period" for the ventral tegmental area, in which early-life stress induces downregulation of orthodenticle homeobox 2 (Otx2) in the ventral tegmental area at P17, followed by long-lasting change in stress-sensitive depression-like behaviours. ${ }^{42,43} \mathrm{We}$ identified the narrow time window of P10-20 as the sensitive period for the lateral habenula - similar to the ventral tegmental area; physically mild chronic stress during P10-20 induced long-lasting alterations in behaviour. Thus, the lateral habenula is thought to have an experience-dependent sensitivity period in the neural circuits that process emotional information during P10-20. The effects of experiences during P10-20 contrast clearly with those in adulthood, in which only intense stress stimulations affect the lateral habenula. ${ }^{7-10,44,45}$

These findings remind us of critical periods in the sensory system, in which experiences modify neuronal circuits. ${ }^{19,31,32}$ In the present study, we classified the maturation of the lateral habenula into 4 stages and found that the lateral habenula is highly plastic during the early-life period of P10-20 in mice; experiences during this period modified PNNs and PV neurons, and resulted in behaviour alterations in adults. Repeated maternal deprivation during P10-20 is a suitable experimental model that helps us understand behavioural disorders as a result of experience-dependent circuit plasticity in the lateral habenula, possibly mimicking child neglect and abuse.

\section{Limitations}

Immobility in the forced swim test has been argued to be a depressive-like behaviour, but an alternative explanation is that the immobility results from learning and memory promoting behavioural adaptations. ${ }^{46-49}$ Further elucidation of the forced swim test is needed.

The roles of lateral habenula PV and PNNs in behavioural alterations are still to be investigated. It is important to understand the mechanism of the "sensitive period" of neuronal circuits in the lateral habenula, and how P10-20 is different from P9 or earlier, or P35 or later. 


\section{Conclusion}

Early-life stress in P10-20 had later effects in adulthood: hyperactivity in the lateral habenula and anxiety or depression, indicating differences in neuronal plasticity between stages of maturation in the lateral habenula. Experiences in early life are essential for the maturation of neuronal circuits in the lateral habenula that underlie emotion.

Acknowledgements: The authors thank Drs. H. Nishijo, H. Nishimaru and A. Uematsu for critical reading of the manuscript and Drs. Y. Takeuchi and M. Kawaguchi for discussions. This research was supported by Kakenhi JP26221004 and 17K17747 (JSPS), grantsin-aid from the Hokuriku Bank, Ltd., the Tamura Science and Technology Foundation, the Toyama Hitozukuri Foundation, and Research Projects from the University of Toyama in Toyama, Japan.

Affiliations: From the Department of Anatomy, Faculty of Medicine, University of Toyama, Toyama, Japan (Nakamura, Kurosaki, Kanemoto, Ichijo); and the Department of Pathology Faculty of Medicine, University of Toyama, Toyama, Japan (Sasahara).

Competing interests: None declared.

Contributors: T. Nakamura and H. Ichijo designed the study. T. Nakamura, K. Kurosaki, M. Kanemoto and M. Sasahara acquired the data, which T. Nakamura and K. Kurosaki analyzed. T. Nakamura and H. Ichijo wrote the article, which K. Kurosaki, M. Kanemoto, and M. Sasahara reviewed. All authors approved the final version to be published and can certify that no other individuals not listed as authors have made substantial contributions to the paper.

Content licence: This is an Open Access article distributed in accordance with the terms of the Creative Commons Attribution (CC BY-NC-ND 4.0) licence, which permits use, distribution and reproduction in any medium, provided that the original publication is properly cited, the use is noncommercial (i.e., research or educational use), and no modifications or adaptations are made. See: https:/ / creativecommons.org/licenses/by-nc-nd/4.0/

\section{References}

1. Hikosaka O, Sesack SR, Lecourtier L, et al. Habenula: crossroad between the basal ganglia and the limbic system. J Neurosci 2008; 28:11825-9.

2. Aizawa H, Amo R, Okamoto H. Phylogeny and ontogeny of the habenular structure. Front Neurosci 2011;5:138.

3. Proulx CD, Hikosaka O, Malinow R. Reward processing by the lateral habenula in normal and depressive behaviors. Nat Neurosci 2014;17:1146.

4. Mizumori SJ, Baker PM. The lateral habenula and adaptive behaviors. Trends Neurosci 2017;40:481-93.

5. Yang Y, Wang H, Hu J, et al. Lateral habenula in the pathophysiology of depression. Curr Opin Neurobiol 2018;48:90-6.

6. Kang S, Li J, Zuo W, et al. Ethanol withdrawal drives anxiety-related behaviors by reducing M-type potassium channel activity in the lateral habenula. Neuropsychopharmacology 2017;42:1813.

7. Li B, Piriz J, Mirrione M, et al. Synaptic potentiation onto habenula neurons in the learned helplessness model of depression. Nature 2011;470:535.

8. Li K, Zhou T, Liao L, et al. $\beta$ CaMKII in lateral habenula mediates core symptoms of depression. Science 2013;341:1016-20.

9. Yang Y, Cui Y, Sang K, et al. Ketamine blocks bursting in the lateral habenula to rapidly relieve depression. Nature 2018;554:317-22.

10. Winter C, Vollmayr B, Djodari-Irani A, et al. Pharmacological inhibition of the lateral habenula improves depressive-like behavior in an animal model of treatment resistant depression. Behav Brain Res 2011;216:463-5.

11. Morris JS, Smith KA, Cowen PJ, et al. Covariation of activity in habenula and dorsal raphe nuclei following tryptophan depletion. Neuroimage 1999;10:163-72.
12. Li M, D'arcy C, Meng X. Maltreatment in childhood substantially increases the risk of adult depression and anxiety in prospective cohort studies: systematic review, meta-analysis, and proportional attributable fractions. Psychol Med 2016;46:717-30.

13. Rehan W, Antfolk J, Johansson A, et al. Experiences of severe childhood maltreatment, depression, anxiety and alcohol abuse among adults in Finland. PLoS One 2017;12:e0177252.

14. MacQueen GM, Ramakrishnan K, Ratnasingan R, et al. Desipramine treatment reduces the long-term behavioural and neurochemical sequelae of early-life maternal separation. Int J Neuropsychopharmacol 2003:6:391-6.

15. Aisa B, Tordera R, Lasheras B, et al. Cognitive impairment associated to HPA axis hyperactivity after maternal separation in rats. Psychoneuroendocrinology 2007;32:256-66.

16. Millstein RA, Holmes A. Effects of repeated maternal separation on anxiety-and depression-related phenotypes in different mouse strains. Neurosci Biobehav Rev 2007;31:3-17.

17. Molet J, Maras PM, Avishai-Eliner S, et al. Naturalistic rodent models of chronic early-life stress. Dev Psychobiol 2014;56:1675-88.

18. Tchenio A, Lecca S, Valentinova K, et al. Limiting habenular hyperactivity ameliorates maternal separation-driven depressive-like symptoms. Nat Commun 2017;8:1135.

19. Gordon JA, Stryker MP. Experience-dependent plasticity of binocular responses in the primary visual cortex of the mouse. J Neurosci 1996;16:3274-86

20. Ichijo H, Hamada M, Takahashi S, et al. Lateralization, maturation, and anteroposterior topography in the lateral habenula revealed by Zif268/Egr1 immunoreactivity and labeling history of neuronal activity. Neurosci Res 2015:95:27-37.

21. Tamamaki N, Yanagawa Y, Tomioka R, et al. Green fluorescent protein expression and colocalization with calretinin, parvalbumin, and somatostatin in the GAD67-GFP knock-in mouse. J Comp Neurol 2003;467:60-79.

22. Taniguchi $\mathrm{H}, \mathrm{He} \mathrm{M}, \mathrm{Wu} \mathrm{P}$, et al. A resource of Cre driver lines for genetic targeting of GABAergic neurons in cerebral cortex. Neuron 2011;71:995-1013.

23. Abe T, Kiyonari H, Shioi G, et al. Establishment of conditional reporter mouse lines at ROSA26 locus for live cell imaging. Genesis 2011;49:579-90.

24. Guide for the care and use of laboratory animals. Washington (DC): National Research Council; 2010.

25. Paxinos G, Franklin KB. Paxinos and Franklin's the mouse brain in stereotaxic coordinates. Vol. 2. Cambridge (MA): Academic Press; 2004.

26. Ohayon S, Avni O, Taylor AL, et al. Automated multi-day tracking of marked mice for the analysis of social behavior. I Neurosci Methods 2013;219:10-9.

27. Cullinan WE, Ziegler DR, Herman JP. Functional role of local GABAergic influences on the HPA axis. Brain Struct Funct 2008; 213:63.

28. Lodge DJ, Behrens MM, Grace AA. A loss of parvalbumin-containing interneurons is associated with diminished oscillatory activity in an animal model of schizophrenia. J Neurosci 2009;29:2344-54.

29. Villalobos $\mathrm{CA}, \mathrm{Wu} \mathrm{Q}$, Lee $\mathrm{PH}$, et al. Parvalbumin and GABA microcircuits in the mouse superior colliculus. Front Neural Circuits 2018;12:35.

30. Zhao C, Eisinger B, Gammie SC. Characterization of GABAergic neurons in the mouse lateral septum: a double fluorescence in situ hybridization and immunohistochemical study using tyramide signal amplification. PLoS One 2013;8:e73750.

31. Sugiyama S, Di Nardo AA, Aizawa S, et al. Experience-dependent transfer of Otx2 homeoprotein into the visual cortex activates postnatal plasticity. Cell 2008;134:508-20.

32. Miyata S, Komatsu Y, Yoshimura Y, et al. Persistent cortical plasticity by upregulation of chondroitin 6-sulfation. Nat Neurosci 2012;15:414.

33. Ribic A, Crair MC, Biederer T. Synapse-selective control of cortical maturation and plasticity by parvalbumin-autonomous action of SynCAM 1. Cell Rep 2019;26:381-93.e6.

34. Kjellén L, Lindahl U. Proteoglycans: structures and interactions. Annu Rev Biochem 1991;60:443-75.

35. Webster JF, Vroman R, Balueva K, et al. Disentangling neuronal inhibition and inhibitory pathways in the lateral habenula. Sci Rep 2020;10:8490.

36. Rosenfeld P, Wetmore JB, Levine S. Effects of repeated maternal separations on the adrenocortical response to stress of preweanling rats. Physiol Behav 1992;52:787-91. 
37. Blume SR, Padival M, Urban JH, et al. Disruptive effects of repeated stress on basolateral amygdala neurons and fear behavior across the estrous cycle in rats. Sci Rep 2019;9:12292.

38. Laine MA, Sokolowska E, Dudek M, et al. Brain activation induced by chronic psychosocial stress in mice. Sci Rep 2017;7:15061.

39. Skórzewska A, Lehner M, Wisłowska-Stanek A, et al. The effect of chronic administration of corticosterone on anxiety-and depressionlike behavior and the expression of GABA-A receptor alpha-2 subunits in brain structures of low-and high-anxiety rats. Horm Behav 2014;65:6-13.

40. Yarom O, Maroun M, Richter-Levin G. Exposure to forced swim stress alters local circuit activity and plasticity in the dentate gyrus of the hippocampus. Neural Plast 2008.

41. Saxe MD, Battaglia F, Wang JW, et al. Ablation of hippocampal neurogenesis impairs contextual fear conditioning and synaptic plasticity in the dentate gyrus. Proc Natl Acad Sci U S A 2006;103: 17501-6.

42. Peña CJ, Kronman HG, Walker DM, et al. Early life stress confers lifelong stress susceptibility in mice via ventral tegmental area OTX2. Science 2017;356:1185-8.
43. Peña CJ, Nestler EJ, Bagot RC. Environmental programming of susceptibility and resilience to stress in adulthood in male mice. Front Behav Neurosci 2019;13:40.

44. Yang LM, Hu B, Xia YH, et al. Lateral habenula lesions improve the behavioral response in depressed rats via increasing the serotonin level in dorsal raphe nucleus. Behav Brain Res 2008;188:84-90.

45. Seo JS, Zhong P, Liu A, et al. Elevation of p11 in lateral habenula mediates depression-like behavior. Mol Psychiatry 2018;23:1113.

46. De Pablo JM, Parra A, Segovia S, et al. Learned immobility explains the behavior of rats in the forced swimming test. Physiol Behav 1989:46:229-37.

47. Campus P, Colelli V, Orsini C, et al. Evidence for the involvement of extinction-associated inhibitory learning in the forced swimming test. Behav Brain Res 2015;278:348-55.

48. Molendijk ML, de Kloet ER. Immobility in the forced swim test is adaptive and does not reflect depression. Psychoneuroendocrinology 2015;62:389-91.

49. de Kloet ER, Molendijk ML. Coping with the forced swim stressor: towards understanding an adaptive mechanism. Neural Plast 2016; 2016:6503162. 